\section{Suicide: the leading cause of maternal death*}

MARGARET OATES report. However, in future ONS Linkage will pursue non-reported cases.

\section{Suicide}

Of the maternal deaths reported to the CEMD, $12 \%$ were due to psychiatric causes and $10 \%$ to suicide. These and other causes of psychiatric death are shown in Table 1.

Initially, suicide appears as the second

\section{THE CONFIDENTIAL ENQUIRIES INTO MATERNAL DEATHS}

The 1997-1999 report of the Confidential Enquiries into Maternal Deaths (CEMD, 2001) identifies suicide as the leading cause of maternal death. The CEMD have always included suicide and much of the influential research on suicide and gender has used their data. However, a psychiatrist has participated only in the last two reports, when psychiatric deaths have been analysed separately.

\section{MATERNAL DEATHS}

A maternal death follows a registerable live birth or stillbirth at or more than 24 weeks of gestation. Maternal deaths are classified as direct (e.g. haemorrhage), indirect (e.g. cardiac) or coincidental (e.g. accidents). They can occur in pregnancy, within 42 days of delivery (early) or after 42 days to 1 year (late). Caution is needed when comparing international maternal mortality late maternal deaths inflates the UK rates.

In the UK, maternal mortality rates began to decline sharply in the 1930s. The direct rate has decreased over the years of the CEMD. The indirect rate has increased as a result of improved case ascertainment.

\section{DEATHS FROM PSYCHIATRIC CAUSES}

A death is described as being due to a psychiatric cause if it would not have occurred in the absence of psychiatric disorder. The majority are due to suicide, with a minority due to substance misuse (mainly accidental overdoses of heroin) and a few other causes (e.g. adverse drug reactions and homicide).

* This editorial is an adapted version of chapter II of Why Mothers Die (CEMD, 200I). rates because the inclusion of indirect and
All psychiatric deaths are classified as indirect. This may seem inappropriate when suicide is due to a severe post-partum depressive illness or puerperal psychosis.

During 1997-1999 there were 2123614 maternities and 242 deaths from both direct and indirect causes reported to the CEMD, which gives a maternal mortality rate of 11.4 per 100000 . By adding coincidental and late deaths, the total becomes 378 maternal deaths, which gives an overall maternity mortality rate of 17.8 per 100 000. Case ascertainment by the CEMD results in higher figures than with death certificates, which may not record childbirth. During 1997-1999 a pilot study conducted by the Office for National Statistics (ONS) for the CEMD, the 'ONS Linkage Study', further improved case ascertainment by linking deaths with births in the previous year. This revealed a further 200 deaths. Predictably, the majority were late and all were indirect causes of death. Insufficient details were available on these additional cases to include them in this

\section{FINDINGS OF THE CONFIDENTIAL ENQUIRIES INTO MATERNAL DEATHS} leading cause of maternal death (thromboembolism is the lead cause). However, when the ONS cases are added a further 59 women died from psychiatric causes (48 suicides and 11 by substance misuse). Suicide then emerges as the leading cause of maternal death.

\section{Violent methods}

In keeping with the 1994-1996 CEMD report (Department of Health, 1998) most died violently, mainly by hanging or jumping. Only three women died from an overdose of medication. Despite the inability to include the ONS cases, the method of suicide was known and the findings of the CEMD in this respect would not have been altered by their inclusion.

Previous studies consistently have found gender differences in the methods of suicide. Women are less likely to die violently and more likely to die from an overdose (Hawton, 2000; Schapira et al, 2001). The findings of both the CEMD and the ONS Linkage Study stand in contrast to this. The relative social advantage and higher education of the women who died from suicide confirms previous findings (Qin et al, 2000). Female suicide is less associated with unemployment, adversity, single status and divorce.
Table I Time of death in relation to pregnancy from psychiatric causes, UK 1997-1999

\begin{tabular}{lcccc}
\hline & Pregnant $(n)$ & Early' $^{\prime}(n)$ & Late $^{2}(n)$ & Total $(n)$ \\
\hline Suicide & 6 & 7 & 15 & 28 \\
Overdose of illicit drugs & 2 & 0 & 4 & 6 \\
Other & - & 1 & 1 & 2 \\
$\quad$ Pulmonary embolism & - & 1 & - & 1 \\
Murder & 3 & - & 1 & 4 \\
Alcohol-related & - & 1 & - & 1 \\
Adverse drug reaction & 11 & 10 & 21 & 42 \\
Total & & & &
\end{tabular}

I. Within 42 days of delivery.

2. More than $\mathbf{4 2}$ days after delivery. 


\section{The protective effect of maternity?}

The suicide rate is lower than in men, decreasing at a greater rate and thought to be lowest of all in pregnancy and in the two years following birth (Hawton, 2000; Qin et al, 2000), leading to the widespread belief in the 'protective effect of maternity'. The 1997-1999 suicide rate is 2 per 100000 maternities. This contrasts with the rate among all women of 3.4 per 100000 (Schapira et al, 2001). However, it may be that subgroups of women are at an elevated risk of suicide in defined time periods during pregnancy and following birth (Appleby, 1996). The CEMD findings would support this view.

The incidence of post-partum mental illness, admission to psychiatric hospital following delivery and contact with psychiatric services is well established (Kendell et al, 1987; Oates, 1996). It is possible to estimate the number of maternities likely to have suffered from puerperal psychosis, the number in contact with psychiatric services and the number admitted to hospital during 1997-1999. This gives an estimated suicide rate for puerperal psychosis of 2 per 1000 sufferers and of 0.3 per 1000 for women referred to psychiatric services following childbirth.

\section{The importance of serious mental illness}

In $86 \%$ of psychiatric maternal deaths it was possible to make a probable diagnosis: $56 \%$ of psychiatric deaths and $68 \%$ of suicides appear to have been suffering from a serious mental illness (psychosis or severe depressive illness).

No diagnosis was possible for the ONS cases; therefore, the finding that the majority of the women who died from suicide were seriously ill may not be maintained if these missing cases could be included. Nonetheless, all the early suicides were suffering from serious mental illness. There were no early deaths in the ONS Linkage Study. All of the early suicides were living in comfortable social circumstances and had an early, abrupt-onset psychotic illness, usually within days of childbirth.

Of the suicides reported to the CEMD, $46 \%$ had previous contact with psychiatric services. All of those who died from substance misuse had previous contact with substance misuse services. Of the women with a previous history, half had a psychiatric admission following a previous childbirth. This is in keeping with our knowledge of post-partum illness.

\section{Awareness of the risk of recurrence}

There were very few cases where either the psychiatric or maternity services had been aware of the risk of recurrence following delivery, and even fewer where management plans had been put in place. A previous history of serious mental illness, post-partum or otherwise, poses a risk of recurrence following childbirth of between 1 in 3 and 1 in 2 (Wieck et al, 1991). Despite this, reports to the coroner suggest that the outcome seems to have taken everyone by surprise.

\section{Problems with the term 'postnatal depression'}

The CEMD were surprised to find that in those few cases where a previous postpartum psychiatric history had been recorded in the maternity notes it was described as postnatal depression and not a psychosis requiring in-patient treatment. The CEMD speculate that this diminished the seriousness of the condition and its need for proactive management.

\section{A CASE FOR IMPROVED DETECTION OR IMPROVED MANAGEMENT?}

Of all the women who died, $85 \%$ had their psychiatric problems identified and were receiving treatment, $46 \%$ of the suicides were in contact with psychiatric services and the majority of those with substance misuse were in contact with substance misuse services. In only three cases were women not receiving any care.

\section{IMPLICATIONS FOR PRACTICE}

The profile of women who died from suicide seems different to that of men and non-childbearing women. The woman most likely to kill herself is one who has had a previous experience of hospitalised mental illness without her baby and who suffers from a severe mental illness with an early onset following childbirth, who is older and free from social adversity and whose act of deliberate self-harm is violent.

All those working in obstetrics and psychiatry need to be reminded that a minority of women will suffer from a severe illness of sudden onset in the early puerperium that requires specialist management. They also need to be reminded of the high rate of recurrence after subsequent pregnancies and that the timing and severity of these illnesses are likely to be as before. Women who have suffered from severe non-postpartum illness also face a high risk of recurrence. At the booking clinic, asking specifically about the history of serious mental illness is as essential as asking questions about diabetes and epilepsy.

Few psychiatric events are as predictable as a post-partum recurrence and they come with 9 months' warning. Management plans and close surveillance in the early post-partum period are essential.

\section{THE ROLE OF SPECIALIST SERVICES}

All the women who died from suicide and who had been admitted at any time following the birth of a baby had been admitted to a general psychiatric unit and separated from their infant. The CEMD speculated on the contribution that this made to the suicide. No death occurred in a woman admitted at any time to a mother and baby unit.

Recommendations informed from the CEMD key findings form the basis for clinical risk management standards for maternity services issued by the Clinical Negligence Scheme for Trusts (CNST, 2002). It is of concern that in many localities the skills and resources to meet these standards may not be in place. Similar recommendations have been made in the past by the Royal College of Psychiatrists (2000) and the NHS in Scotland (2001) and are likely to be included in the forthcoming Children and Women's Health National Service Framework.

\section{RECOMMENDATIONS}

(a) Protocols for the management of women at risk of a serious mental illness following delivery should be in place in every maternity service.

(b) Enquiries about previous psychiatric history should be made routinely at the antenatal booking clinic.

(c) The term 'postnatal depression' should not be used as a term for all types of psychiatric disorder. 
(d) Women who have a past history of serious psychiatric disorder (postpartum or non-post-partum) should be assessed by a psychiatrist in the antenatal period with regard to the high risk of recurrence following delivery.

\section{CONCLUSIONS}

Suicide following childbirth is rare but the rates are higher than previously thought. Some women died despite exemplary care. For others, it is impossible to know whether factors that appear to contribute to the death are also to be found in women who do not die. However, a quarter of suicides might not die if their high risk of postpartum recurrences are identified. For some, better management of their acute post-partum illness might improve the outcome.

Hopefully, these recommendations will save some lives in the future and improve the care of women with serious mental health problems associated with childbirth.

\section{DECLARATION OF INTEREST}

M.O. is a member of the Confidential Enquiries into Maternal Deaths and author (on behalf of the CEMD) of Chapter 11

MARGARET OATES, FRCPsych, Department of Psychiatry, University Hospital, Queen's Medical Centre, Clifton Boulevard, Nottingham NG7 2UH, UK. E-mail: margaret.oates@nottingham.ac.uk

(First received 5 September 2002, final revision 17 April 2003, accepted 6 May 2003)

'Deaths from Psychiatric Causes', upon which this editorial is based (see http:// www.doh.gov.uk/cmo/mdeaths.htm).

\section{ACKNOWLEDGEMENTS}

Thanks to Dr Gwyneth Lewis, Director and Editor of the CEMD report. We also owe a debt of gratitude to the late Professor R. Kumar, the first psychiatrist to be a member of the CEMD.

\section{REFERENCES}

Appleby, L. (1996) Suicide behaviour in childbearing women. International Review of Psychiatry, 8, 107-II5.

CEMD (Confidential Enquiries into Maternal Deaths) (2001) Why Mothers Die 1997-1999. London: Royal College of Obstetricians and Gynaecologists.

CNST (Clinical Negligence Scheme for Trusts) (2002) Clinical Risk Management Standards for Maternity Services. London: NHS Litigation Authority/Willis.

Department of Health (1998) Deaths from psychiatric causes suicide and substance abuse. In Why Mothers Die: Report on Confidential Enquiries into Maternal Deaths in the United Kingdom 1994-1996. London: Stationery Office.
Hawton, K. (2000) Sex and suicide: gender differences in suicidal behaviour. British Journal of Psychiatry, $\mathbf{I 7 7}$ 484-485.

Kendell, R. E., Chalmers, K. C. \& Platz, C. (1987) Epidemiology of puerperal psychoses. British journal of Psychiatry, 150, 662-673.

NHS in Scotland (200I) Framework for Maternity Services in Scotland. NHS in Scotland.

Oates, M. (1996) Psychiatric services for women following childbirth. International Review of Psychiatry, $\mathbf{8}$, 87-98.

Qin, P., Mortensen, P. B., Agerbo, E., et al (2000) Gender differences in risk factors for suicide in Denmark. British Journal of Psychiatry, 177, 546-550.

Royal College of Psychiatrists (2000) Perinatal Maternal Mental Health Services. Council Report CR88. London: Royal College of Psychiatrists.

Schapira, K., Linsley, K. R., Linsley, A., et al (200I) Relationship of suicide rates to social factors and availability of lethal methods. Comparison of suicide in Newcastle upon Tyne 1961-1965 and 1985-1994. British Journal of Psychiatry, I78, 458-464.

Wieck, A., Kumar, R., Hirst, A. D., et al (1991) Increased sensitivity of dopamine receptors and recurrence of affective psychosis after childbirth. $B M$ J $303,613-616$. 